\title{
NOTAS DE CLINICA THERAPEUTICA
}

\section{Prof. CELESTINo bourroul}

\section{1.a Serie: APPARELHO RESPIRATORIO}

\section{I) Pneumonia lobar aguda}

Definição: Pneumococcia pulmonar, geralmente lobar, pelo Pneumococco de Talamon-Frankel.

Etiologia: A frigore commummente.

Symptomas: Febre alta de vez $-39 .^{\prime}$ a $400^{\prime}$. (Toda a febre alta d'emblée, com um estado pulmonar, - resfriado, tosse - deve trazer a ideia de pneumonia ou broncho-pneumonia, - esta nas creanças principalmente)

Escarros fibrinosos, pegajosos, côr de ferrugem ou de tijolo, adlerentes ao fundo do vaso. Sub-massicez; bronchophonia; au. gmento das vibrações vocaes, sopro tubario. Tosse. Pontada. Calafrio, ás vezes unico : intenso. Na pneumonia central, os signaes physicos fodem falltar: attentar, entíio, para os geraes - febre alta, etc.

$\mathrm{Na}$ pneumonia dos velhos e deprimidos, a febre póde ser baixa.

Os estertores crepitante do inicio du molestia são tão raros e fugazes que não têm valor diagnostico. Localização geralmente, basal; a apical é mais frequente nos velhos, crianças e alcoolicos, tendo nestes p:ognostic o mais sombrio. Na phase de resolução, estertores sub-crepitantes grossos de retorno.

Complicações: Duração -7 a 10 dias; se a molestia dura mais de 10 dias, pensar, então, em complicações: Pleurisia suppurada; resolução demorada na induração e carnificação; fócos multi. plos ou successivos (pneumonia migratoria, dupla); suppuração (hepatisação cinzenta - escarros escuros, de calda de ameixas pretas); ou em localizações outras como meningite, endocardite, pericardite, abcessos, etc. Urina carregada, vermelha, rica em uréa e pobre em chloretos. Terminação normal por crise, acompanhada de suores profusos.

$\mathrm{Na}$ pneumonia arrastada (inđuração, carnificação, fibrose, suppuração), o exame radiologico mostra sombra em forma conica, de cunba, com base peripherica e apice olhando para o hilo, que não deve ser tida por pleurisia interlobar (punç̧ão negatíva)

Nas cacos duvidosos é bom punccionar, pela frequencia das complicações pleuraeミ, sobretudo nas creanças. 
Broncho-pneumonia. - Creanças. - Nestas até $10-13$ annos, a pneumonia lo'bar massiça é mais rara, tendo antes uma localisação lobular (broncho-pneumonia) A respiração pueril, rude por natureza, difficulta nas creanças o diagnostico do sopro tuba. rio. Attender, ahi, ao caracter mais superficial e soproso deste; aos fócos multiplos; á sub-massicez; e sobretudo ao estado geral máu febre, dyspnéa, pallidez, tosse dyspneica caracteristica - como se ow doeñtinhos quizessem escarrar a cada passo a sua tosse.

\section{TRATAMENTO:}

Camphora - Oleo camphorado a 25 o|o em alta dóse. Adultos: $10 \mathrm{cc}$. $(2,5 \mathrm{gr}$ de camphora $)$ de manhã e $10 \mathrm{cc}$. á noite. Noś casos graves: $10 \mathrm{cc}$. de 8 em 8 horas, até 30 cc. por dia. A camphora, além de sua acção tonica cardio-vascular, age sobre os pulmões, eliminando-se pela arvore respiratoria (halito com cheiro de camphora)

Digitalis - Aguentar, desde o começo, o coração com a dugitalis, sobretudo nos doentes de meia edade, deprimidos, alcoolicos, meiopragicos e velhos. A digitalina na dóse de $1 / 5$ de milligr. ( $X$ gottas da digitalina de Petit-Mialhe ou Nativelle) de 'manhã, e se preciso á noite. Digaleno - XX a XXX gottas de manhã e á noite; ou 1 a 2 empolas em injecção. A tintura de digitalis, em vista da riqueza duvidosa das folhas não merece confiança.

Pituitrina Adrenalina - Tonificar o systema circulatorio peripherico (paralysia ou parese vascular) com os vaso-constrictores pituitrina (1 empola) e com a adrenalina ( $P$ \& D.), em injecções, de $1 \mid 4$ a $1 \mid 2$ milligr., até 2 a 3 milligr por dia.

Medicação especifica. Soro. Vaccina - Tentar a medicação especifica - soro antipneumococcico polyvalente e vaccinas polyvalentes; se possivel e se houver tempo - a autovaccina.

Quinina - Corrigir o estado infeccioso - A quinína (bromhydrato ou bi-chlohydrato) - $1 \mid 2 \mathrm{gr}$ a $0, \mathrm{gr} 60$ - capsulas de 0,15 a 0,20 - 3 vezes por dia.

Colloidaes - Metaes colloidaes - O electrargol de Clin dá bons resultados nas creanças. Nunca injectar colloidaes nas veias reacções fortissimas, febris e colloidoclasicas, ás vezes fataes.

O cobre e ouro colloidaes, associado $_{S}$ ou não ao formiato de quinina. em injecção (Ionase de Orlando Rangel v g ) são indicados principalmente nas formas grippaes.

Febre. Compressas frias - Moderar a febre pelos envoltorios frios do thorax (compressas de agua fria), um de 3 em 3 horas e durante $1 \mid 2$ a 1 hora, isto é, até que a compressa se apresente 
quente ou que o doente tenha sensação de calor Se a temperatura depois da primeira compressa se mantiver alta podem_se applicar 3 compressas em seguida, medeiando um intervallo de $1 \mid 4$ de hora entre uma e outra.

Nos doentes sensiveis á agua fria, começar com compressas humidas de partes eguaes de alcool e agua, ou ainda com compresas mornas.

Lençol molhado. Banhos - Se a temperatura não baixar $40^{\circ} .5,41^{\circ}$. - lençol molhado em todo o corpo ou mesmo banhos mornos, na temperatura inicial de de 37: e baixando-se decrescentemente a $34 .^{\circ}$ e $300^{\circ}$.

Antithermicos - Os antithermicos carecem ser dados com moderação, não mais de $1 \mid 2$ gr por dia, - pequenas dóses de aspirina, pyramiđo ou cryogenina - puros ou associados á quinina, em vista da acção deprimente e da sudorose que provocam.

Capsulaś do typo das antigrippaes, como:

N.o 1

Bromhydrato de quinina $0: 20$

Aspirina $\quad 0,20$

Pyramido ou Cryogenina 0,10

Pós de Dover $\quad 0,05$

Cafeina $\quad 0,05$

Para 1 capsula. Tome 3 por dia.

Pontadas. Sinapismos - Cataplasmas synapisadas. As ponta. das. pleurite, são combatidas com as cataplasmas sinapisadas - papas de farinha de mandioca ou de linhaça polvilhadas de farinha de mostarda ou 2/3 partes de linhaça com $1 \mid 3$ de mostarda; ou ventoisas seccas ou escarificadas Deixar as ventosas escarificadas para ultimo lugar, pois a pelle ferida não supportaria nem outro topico.

Fmbrocações - Fricções ou compressas de salicylato de methyla, chloroformio ou de essencia de therebentina podem ser applicados loco dolenti.

Pontas de fogo - Não se indicam nas formas agudas mas nas formas arrastadas - pleurite, induração - pela reacção congestiva
que provocam.

Vesicatorios - 0 vesicatorio de cantharidas, muito empregado antigamente nas pneumonias agudas, cahiu em desuso, pelo perigo da nephrito centharidiana. Nos casos muito especiases de resolução demorada. merecem ser lembrados os revulsivos de thapsia ou oleo de croton, pela inflammação substitutiva e processos leucosytarics de defesa. 
Expectorantes - O bloco pneumonico soffre uma resolução mais interna pelos leucocytos do que externa pela expectoração Os expectorantes ammoniacaes indicam_se no accumulo de catarrho com expectoração difficil e com parese dos bronchios.

No. 2

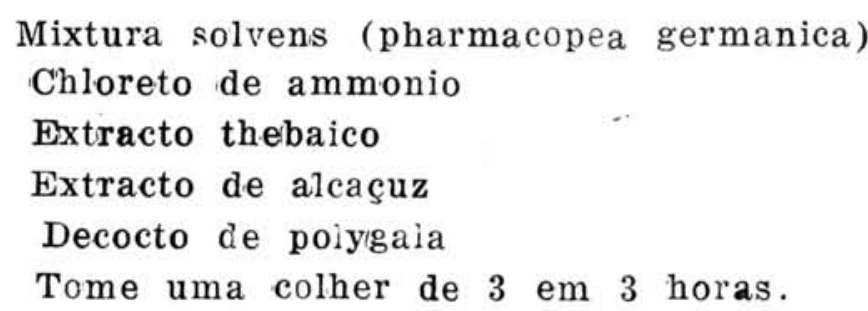

No. 3

Chloreto de ammonio $3 \mathrm{gr}$

Oxymel scillitico

Xarope de codeina

(ou Xarope de belladona)

Infuso de ipeca a $1 \mathrm{o}$

(ou de polygala ou de atnica)

Tome anı colher de 3 em 3 horas.

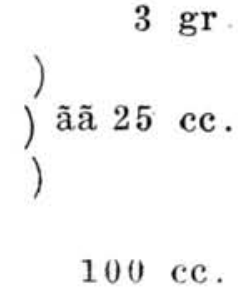

Eronchoplegia. Congestãc - Nas formas congestivas e bronchoplegicas a ipeca ou emetina; a estrychnina até 5 milligrs. por dia em doses fraccionadas; a pituitrina, 1 a 2 empolas por dia; a adrenalina, 2 a 3 milligr em injecção, em doses fraccionadas de $1 \mid 4$ a $1 \mid 2$ milligr.

Antimoniaes - Nas bronchorrhéas e formas congestivas, convem ás vezes os antimoniaes como modificadores - kermes mineral $(0,10$ a 0,20$)$, oxydo branco de antimonio, $1 \mid 2$ a $1 \mathrm{gr}$

Alccol - Na pneumonia dos alcoolicos e deprimidos dar aguardente, cognac ou vinho do Porto ou poção de Todd ou mistura de Stokes.

Ogygenio. Abcesso de fixação - Nos casos graves, asphyxicos, fazer inhalações de oxygenio constantemente (tubo de oxygenio); e injecção de 1 a $2 \mathrm{cc}$. de essencia de therebentina, para o abcesso de fixação, na parte externa da coxa.

Formas protra ${ }^{n}$ idas. Creosotados - Nos processos torpidos os areosotados-thiocol, $1 \mid 2$ a 1 gr. e os balsamicos-terpina $1 \mid 2 \mathrm{gr}$. s€ indicam .

As injecçõ ss derivadas de formula:

$\begin{array}{ll}\text { Gaiacol } & 0,05 \\ \text { Menthol } & 0,05\end{array}$




$\begin{array}{ll}\text { Eucalyptol. } & 0.05 \\ \text { Iodoformio } & 0,01 \\ \text { Phenol. } & 0,01\end{array}$

Oleo camphorado a 25 o|o q. b. para 2 cc.

Uma empola por dia ou cada 2 dias.

Iodados. Arsenicaes - Xarope iodotannico puro ou associado ao iodeto de calcio (1|2 a $1 \mathrm{gr})$

Oleo de figado de bacalhau - Arsenicaes, arrhenal ou cacodylato $(0,05)$ em injecções e o oleo de figado de bacalhau.

\section{II) Broncho-pneumonias agudas}

(Pneumonia lobular)

Definição - Phlogose pulmonar - bronchiolite, alveolĩe (bronchite capillar, catharro suffocante)

Etiologia - Mais circumscripta, mais localizada em um ou mais fócos (forma multipla ou migratoria) não atacando um lobo todo, massiça, como a pneumonia lobar; geralmente secundaria á grippe, pneumococcia, estreptococcia, sarampo, coqueluche, etc.

E' complicação frequente do sarampo e mortal do coqueluche.

Nas creanças até $10-12$ annos a pneumonia toma mais a fórma lobular (broncho-pneumonia) que a lobar

Symptomas - Tosse, dyspnéa - tosse dyspneica (o doentinho escarra a sua tosse) Aqui como na pneumonia lobar, o estado local tem uma assignatura, importante quanto ao diagnostico, no estado geral máu, dyspnéa, pallidez, cyanose, asphyxia.

Febre alta, ein um estado anterior de bronchite deve trazer a suspeita de broncho-pneumonia, principalmente nas creanças.

Fóco isolado ou esparsos, migratorios de sub-massicez, estertores humidos; sopro, menos intenso e franco que o tubario; sibilos, ás vezes é uma tempestade bar ulhenta de sopros, sibilos, estertores e attritos.

\section{TRATAMENTO:}

O mesmo que para a pneumonia, attenđendo-se tambem ao tratamento da infecção causal (grippe, coqueIuche, sarampo)

Vomitorio - $\mathrm{Na}$ bronchite capillar ou catarrho suffocante das creanças, quando o estado geral u permitta, dar vomitorios de ipeca (xarope de ipeca com ipeca em pó a 1 o|o: 1 colherinha de chá de $1 \mid 4$ em $1 \mid 4$ de hora), aç̧ão, descongestionante e desobstruente. 
Adultos, ipeca em pó em capsulas de $1 \mid 2 \mathrm{gr}$ de $1 \mid 4$ em $1 \mid 4$ de hora.

Camphora - A camphura $(0,25$ a 0,50 até $1 \mathrm{gr}$ por dia e por anno de eảade) Convém nas creanças deprimidas tactear a sensibilidade para a camphora, pois o figado insufficiente pode não fixar pelo acido glycuronico a camphora toxica.

Electrargol - O electrargol (1|2 empola) constitue bom tratamento para as creanças.

Quinina - Aristoquina ou euquinina ou tannato de quinina $(0,10$ a 0,20$)$

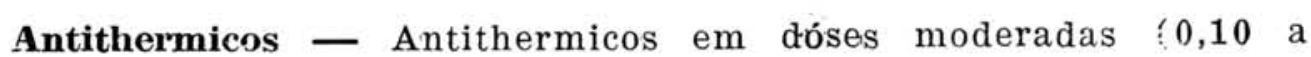
0,20 por anno) associados á quinina.

Digitalis - Digaleno, V gottas por anno de edade.

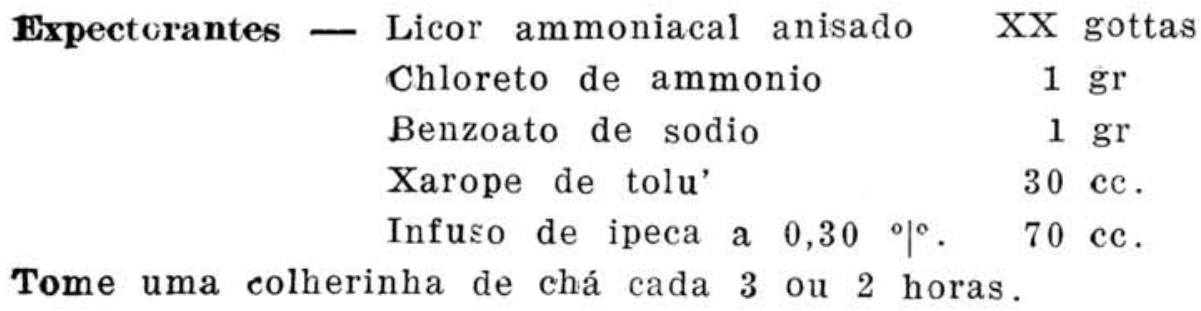

\section{ASSIGNEM A "REVISTA DE MEDIGINA"} ESTRANGEORO . $10 \$ 000$ 